\title{
Research into Methods to Construct Software Engineering Knowledge Database Based on Ontology
}

\author{
$\mathrm{Yu} \mathrm{Yi}$ \\ NanChang Institute of Science \& Technology
}

\begin{abstract}
With the rapid development of Internet technology, the field of software engineering knowledge is constantly updated, and the amount of knowledge has also been rapidly growing. Traditional software engineering knowledge service method, because of lack of semantic retrieval ability, can not meet the growing demand of knowledge, so it has become a new research hotspot to build software engineering knowledge base with semantic service ability. Ontology is a clear specification of the conceptual model, which is widely used in the field of knowledge engineering, information retrieval, and it has a semantic level of concept structure, which can simulate human thinking mode, and become a new direction in the field of knowledge base construction. This paper in view of the current software engineering domain knowledge base in the semantic retrieval is proposed using ontology technology to build knowledge base with semantic features, design with universally applicable value of the ontology based domain knowledge base to construct the model. The purpose is to users to provide semantic reasoning ability of knowledge retrieval in retrieval at the same time to provide users with oriented thinking.
\end{abstract}

Keywords-Software Engineering; Ontology; Knowledge Database; Knowledge Retrival

\section{INTRODUCTION}

China's knowledge engineering is at the stage of rapid development, with the rapid development of information technology, Internet, digital media and other technologies, the construction of knowledge engineering has ushered in tremendous opportunities for development, but also faces many challenges. At present, there are a lot of knowledge databases on the Internet, but because of various reasons, the content is too complex, a lot of useful knowledge is submerged in the vast amount of resources, so that readers can not quickly search.

Software engineering is a core subject in the field of IT, and it has a very important position in the whole field of information technology. With the rapid development of Internet technology, knowledge in the field of software engineering is also rapidly updated, the amount of knowledge has also been rapid growth. In the past, the knowledge of software engineering is mainly through books, periodicals, Internet search, etc., and can not meet the requirements of the Internet era. First, books and periodicals are slow to update their knowledge. Secondly, these knowledge resources can not provide semantic retrieval services. Existing knowledge service has been unable to adapt to the era of large information content of the Internet era background, it is difficult to meet the growing demand for knowledge in the field of software engineering. Based on the above situation, the construction of knowledge service system in the field of software engineering with semantic service is an important task in the construction of knowledge engineering.

This paper in view of the current software engineering domain knowledge base in the semantic retrieval is proposed using ontology technology to build knowledge base with semantic features, design with universally applicable value of the ontology based domain knowledge base to construct the model. The purpose is to users to provide semantic reasoning ability of knowledge retrieval in retrieval at the same time to provide users with oriented thinking. The model consists of three parts: the planning and design of knowledge base, ontology construction and knowledge service.

\section{ESTABLISHMENT OF ONTOLOGY BASED ON}

\section{SOFTWARE ENGINEERING DEVELOPMENT}

This paper in the form of domain ontology framework using the structured method splits mode, the planning stage of domain ontology, domain ontology analysis stage, design stage of domain ontology, domain ontology implementation stage and domain ontology operation stage, each stage has its own independent goals and main tasks, task is usually on the previous stage and put forward the method to solve the question of further concrete, namely the process is in accordance with the software engineering life cycle processes to gradually solve the problem of. In the field of the main analysis phase, according to the specific requirements and objectives of the domain ontology planning stage, the prototype method is used to analyze the results and improve the results. Its architecture is shown in Fig.1. 


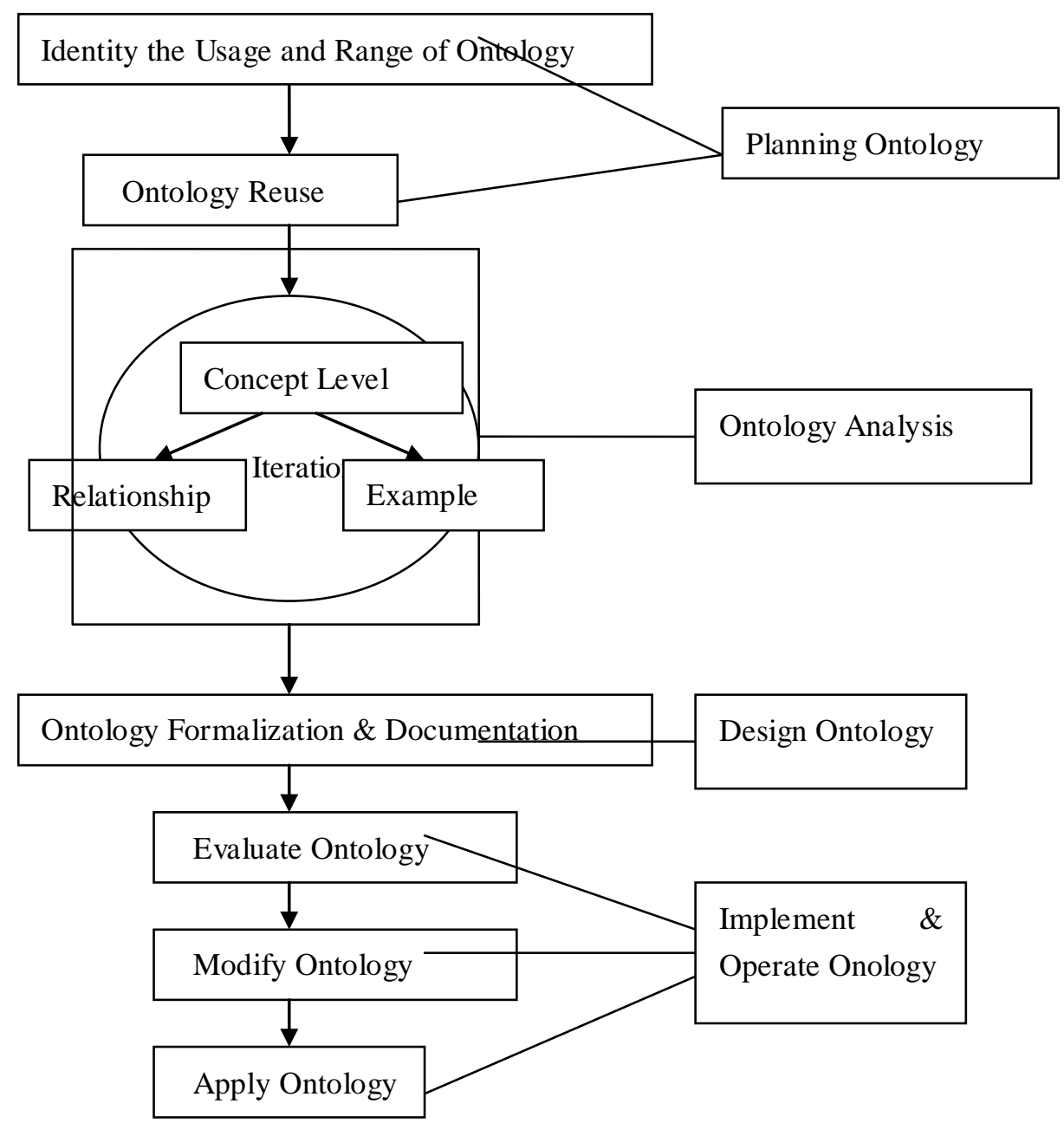

Figure 1. Ontology Construction Framework based on Software Engineering Development

One way to determine the scope of the domain ontology is to design and fill in the ontology of the questionnaire: (1) refine demands; (2) the ability to trace demands; (3) satisfy demands; (4) generate documents; (5) Upgrade.

\section{INTRODUCTION TO ONTOLOGY}

Ontology (Ontology) was originally a concept in the field of philosophy, and it is the world of all kinds of things in the objective description. After being applied to the field of information technology, Gruber has been redefined ". In the ontology, the relationship between the concept and the concept of a knowledge domain is abstracted as a series of formal language, which can be used to identify the communication barrier between human and computer. Ontology is a standard, has been widely recognized, it contains a domain concept and knowledge of a subject, the relationship between domain knowledge and concepts through the terms of the terms of the logical statement to express. Therefore, ontology provides a used to express and communicate some knowledge of Subject Thesaurus and a relation set, set is a collection of vocabulary in these terms.

Under the background of information technology, the ontology can be described by a structured description language which can be identified by computer. According to different application scenarios, ontology description language can be divided into two categories based on artificial intelligence ontology language and ontology language based on Web. With the rapid development of Internet technology, the use of ontology markup language based on Web is becoming more and more extensive, and Fig .2 provides the ontology language stack for W3C. 


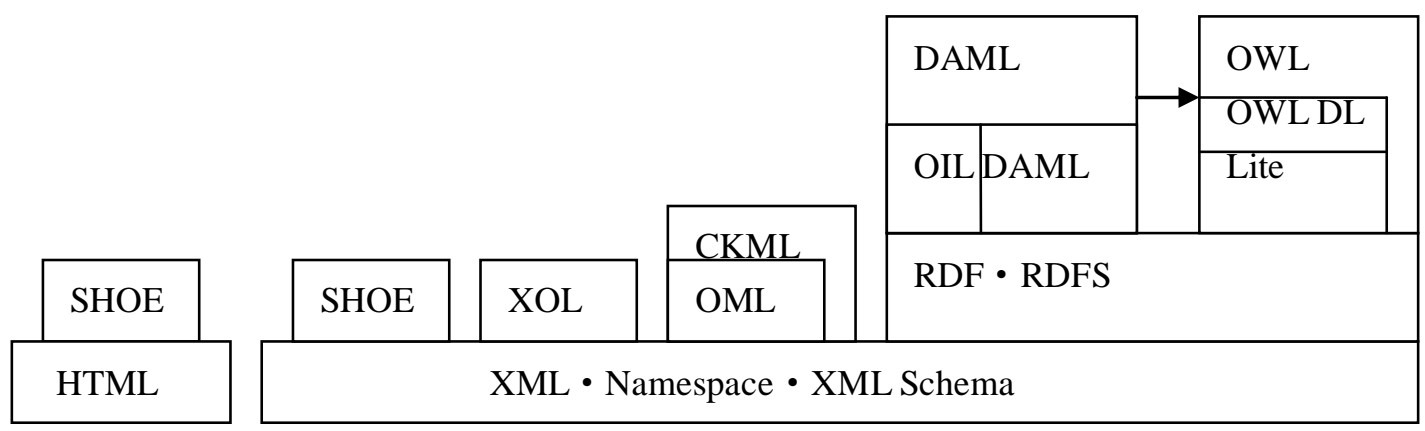

Figure 2. Network Ontology Language Stack

In this paper, the OWL language is used as the ontology description language for the software engineering domain. As a standard ontology description language, is developed from DAML+OIL (Semantic), which is one of the core technologies of W3C OWL (Web). It can describe the relationship between class and class in the Web document and its application. Through the definition of class and instance, a formal knowledge domain is constructed to describe the relationship between class and class, instance and instance.

\section{JENA ONTOLOGY TEChNOLOGY}

Ontology reasoning is an important part of ontology semantic ability, and ontology reasoning is realized by using ontology inference engine. Jena is an open source framework for the semantic web research project of HP labs, based on Java language development. It provides a complete platform, such as RDF, RDFS, OWL, etc., the main functions include RDF, RDFS, OWL and other models to create, syntax analysis, the elements of the model to increase, delete, change, search and other operations, support RDQL ontology query language. Another important feature is the ability to achieve the reasoning based on the user provided by the ontology, including reasoning with the rules of the reasoning. Jena platform includes the following parts:

(1) RDFAPI, which is used to operate texts like OWL for the realization of the document to add, delete, change, check, and create and delete the ontology file.
(2) SPARQL and RDQL query language. ARQ query engine in SPARQL, support RDQL query language and RDQL ontology query language, you can use the SQL language together. The ontology query based on semantic reasoning can be realized by combining Jena's reasoning subsystem.

(3) Inference subsystem. Support RDFS, OWL and other self rule set of reasoning, but also support users to import custom reasoning rule set.

(4) Ontology storage interface. Jena supports the Mysql, PostgreSQL, Oracle and other databases.

In Jena, the framework of ontology model binding Jena ontology language (OntModel), the body into the framework of Jena. OntModel inherits from the Model class in Jena. OntModel Model data collection in the RDF statements and other methods were extended, so that the data objects in the ontology to operate, these objects include: classes, properties, and individual individuals.

The Jena platform can be used as the core module of ontology based semantic retrieval system, and the domain ontology and reasoning rules provided by the semantic retrieval system, and the content of user input to achieve semantic reasoning, and then combined with the SQARQL language to achieve the reasoning results of ontology retrieval. Ontology based semantic retrieval process is shown in Fig .3. 


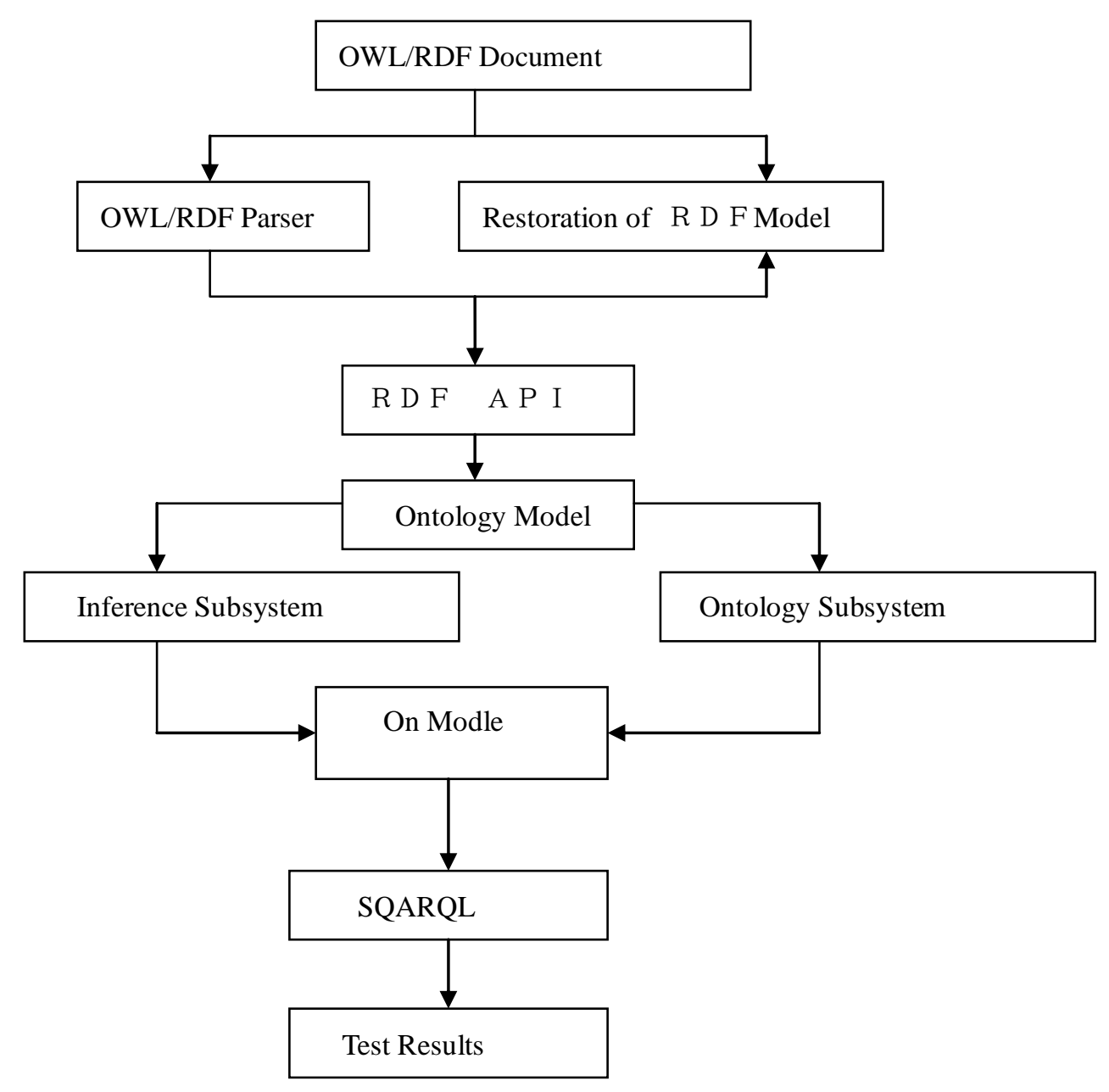

Figure 3. The Process of Semantic Retrieval based on Ontology

\section{V.CONCLUSION}

This paper takes software engineering domain knowledge as an example, and puts forward the construction method and knowledge retrieval method based on ontology technology knowledge base, and verifies the feasibility of the method by using a software engineering knowledge platform. The main research results are as follows:

(1) This paper puts forward a model of ontology knowledge base construction in the field of universal applicability. The model analyzes the common features of the knowledge base in the scientific domain, and puts forward the necessary contents and methods for constructing the ontology knowledge base;

(2) On the method of constructing knowledge relations, this paper analyzes the knowledge structure of Wikipedia, and then maps the knowledge relationship of Wikipedia to the ontology, and then describes them with OWL, and can be displayed by Prot;

(3) On the method of extracting the knowledge attribute, this paper uses the information box (Infobox) of the Wikipedia page to extract the attributes of the knowledge, according to the information structure of the information structure to extract the attributes of knowledge;

\section{REFERENCES}

[1] Maeduche A, Staab S. Ontology Learning for the Semantic Web [J]. IEEE Intelligent Systems, 2011, 16(2): 72-79.

[2] Li S, Li B, Pan W, et al. Approach for building mashup service description ontology automatically $[\mathrm{J}]$. Journal of Chinese Computer Systems, 2011, 32( 9): 1747-1752.

[3] Zhong N, Yao Y, Kakenoto Y. Automatic Construction of ontology from Text Databases[J]. Data Mining, 2011,2: 173-180.

[4] Berriz G F, White J V, King O D, et al. GoFish finds genes with combinations of Gene Ontology attributes.[J]. Bioinformatics, 2003, 19(6):788-789(2).

[5] Gruber T R. A translation approach to portable ontology specifications[J]. Knowledge Acquisition, 1993, 5(2):199-220.

[6] Ashburner M, Ball C A, Blake J A, et al. Gene ontology: tool for the unification of biology. The Gene Ontology Consortium.[J]. Nature Genetics, 2000, 25(1):25-29.

[7] Ashburner M, Ball C A, Blake J A, et al. GOConsortiumAshburner M, Ball CA, Blake JA, Botstein D, Butler H, Cherry JM et al. Gene ontology: tool for the unification of biology. The Gene Ontology Consortium. Nat Genet 25: 25-29[J]. Nature Genetics, 2000, 25.

[8] Ashburner M, Ball C A, Blake J A, et al. Gene Ontology: tool for the unification of biology[J]. Nature Genet, 2000, 25(1):25-29. 
[9] Kalfoglou Y, Schorlemmer W M. Ontology Mapping: The State of the Art. Knowl Eng Rev 18(1): 1-31[J]. Knowledge Engineering Review, 2005, 18

[10] Wache H, Visser U, Stuckenschmidt H, et al. Ontology-based integration of information --- a survey of existing approaches[J]. Ijcai'01 Workshop on Ontologies \& Information Sharing, 2002:108--117.
[11] Noy N F, Mcguinness D L. Ontology Development 101: A Guide to Creating Your First Ontology[J]. And Stanford Medical Informatics, 2001.

[12] Maedche A. Ontology Learning for the Semantic Web.[J]. Intelligent Systems IEEE, 2002, 16(2):72-79. 